\title{
ARTICLE \\ Icariin prevents bone loss by inhibiting bone resorption and stabilizing bone biological apatite in a hindlimb suspension rodent model
}

\author{
Jin-peng $\mathrm{He}^{1}$, Xiu Feng ${ }^{1,2}$, Ju-fang Wang ${ }^{1}$, Wen-gui Shi ${ }^{1,3}, \mathrm{He} \mathrm{Li}^{1}$, Sergei Danilchenko ${ }^{4}$, Aleksei Kalinkevich ${ }^{4}$ and Mykhailo Zhovner ${ }^{4}$
}

\begin{abstract}
Bone loss induced by microgravity is a substantial barrier to humans in long-term spaceflight. Recent studies have revealed that icariin (ICA) can attenuate osteoporosis in postmenopausal women and ovariectomized rats. However, whether ICA can protect against microgravity-induced bone loss remains unknown. In this study, the effects of ICA on a hindlimb suspension rodent model were investigated. Two-month-old female Wistar rats were hindlimb suspended and treated with ICA $\left(25 \mathrm{mg} \cdot \mathrm{kg}^{-1} \cdot \mathrm{d}^{-1}\right.$, i.g.) or a vehicle for 4 weeks $(n=6)$. The bone mass density of the hindlimbs was analyzed using dual-energy X-ray absorptiometry and micro-CT. mRNA expression of osteogenic genes in the tibia and the content of bone metabolism markers in serum were measured using qRT-PCR and ELISA, respectively. The bone mineral phase was analyzed using X-ray diffraction and atomic spectrometry. The results showed that ICA treatment significantly rescued the hindlimb suspension-induced reduction in bone mineral density, trabecular number and thickness, as well as the increases in trabecular separation and the structure model index. In addition, ICA treatment recovered the decreased bone-related gene expression, including alkaline phosphatase (ALP), bone glaprotein (BGP), and osteoprotegerin/receptor activator of the NF-KB ligand ratio (OPG/RANKL), in the tibia and the decreased bone resorption marker TRACP-5b levels in serum caused by simulated microgravity. Notably, ICA treatment restored the instability of bone biological apatite and the metabolic disorder of bone mineral elicited by simulated microgravity. These results demonstrate that ICA treatment plays osteoprotective roles in bone loss induced by simulated microgravity by inhibiting bone resorption and stabilizing bone biological apatite.
\end{abstract}

Keywords: icariin; simulated microgravity; bone loss; hindlimb suspension; apatite; bone resorption

Acta Pharmacologica Sinica (2018) 39:1760-1767; https://doi.org/10.1038/s41401-018-0040-8

\section{INTRODUCTION}

Space environment factors, such as microgravity and ionizing radiation, are serious threats to the health of astronauts, the most prominent of which is bone metabolism derangement [1]. In the astronaut during spaceflight, bone mineral density (BMD) is decreased by $\sim 1 \%$ per month in the proximal femur, which is as much as postmenopausal women on Earth lose in 1 year [2]. Microgravity is considered to be the chief contributor to bone loss, although evidence has shown that ionizing radiation and the magnetic field are involved in induction of bone metabolism diseases $[3,4]$.

Data concerning the effects of microgravity on the human body from long-duration spaceflights are very limited to date. However, in vitro and in vivo models have been developed to reproduce the reduction of bone mass induced by microgravity on Earth $[5,6]$. The hindlimb suspension rodent model, which can produce a lack of load bearing on the hindlimbs and a fluid shift to the head, is extensively used to simulate weightlessness and study aspects of weight-bearing bones in the rodent [7]. By contrast, countermeasures to protect against osteopenia have been studied.
Exercise currently remains the preferred option to counter the effects of microgravity on bone density [8], although its effectiveness is debatable [8, 9]. Pharmacological interventions, which have been successfully used in the clinical treatment of osteoporosis [10, 11], are severely restricted in spaceflights because of the uncertainty of their side effects [12-14].

Icariin (ICA), a flavonoid glucoside (Fig. 1), is the main active ingredient isolated from the genus Epimedium, a traditional Chinese herbal medicine that has been used as a bone protective agent for thousands of years. ICA has many indicated pharmacological and biological activities, such as osteogenic activity, neuroprotection, cardiovascular protection, anti-inflammation, anti-oxidation, and anti-tumor activity $[15,16]$. Previous studies have demonstrated that Epimedium-derived phytoestrogen flavonoids (EPFs) exert beneficial effects on the prevention of bone loss in postmenopausal women, without causing a hyperplasia effect on the endometrium [17]. A recent study reported that treatment of ovariectomized rats with ICA obviously improved the $\mathrm{BMD}$, biomechanical strength, and trabecular bone parameters by mediating Wnt/ $\beta$-catenin pathway [18]. In addition, ICA was

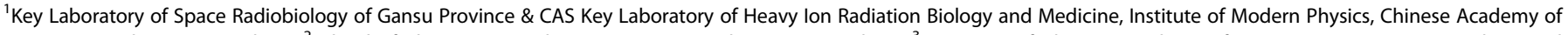

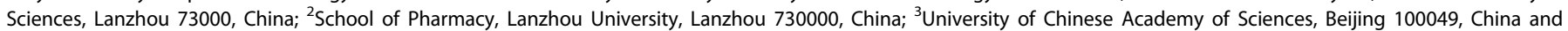
${ }^{4}$ Institute of Applied Physics, National Academy of Sciences of Ukraine, Sumy 40000, Ukraine Correspondence: Ju-fang Wang (jufangwang@impcas.ac.cn)
} 
<smiles>COc1ccc(-c2oc3c(CC=C(C)C)c(OC4O[C@H](CO)[C@@H](O)[C@H](O)[C@H]4O)cc(O)c3c(=O)c2OC2O[C@H](C)[C@@H](O)[C@H](O)[C@H]2O)cc1</smiles>

Icariin (ICA): $\mathrm{C}_{33} \mathrm{H}_{40} \mathrm{O}_{15}$

MW: 676.67

Fig. 1 The chemical structure of icariin

shown to increase cellular proliferation and mineralization in osteoblasts $[19,20]$ and decrease the bone resorption activity in osteoclasts [19, 21]. However, whether ICA can protect against the bone loss caused by microgravity remains unclear.

Cortical or compact bone is a natural composite consisting of $\sim 25 \%$ organic phase, $65 \%$ mineral phase, and $10 \%$ water [22]. Type I collagen is the primary constituent $(90 \%)$ of the organic phase [23]. The mineral phase is crucial for the mechanical properties of bone. It generally consists of poorly crystallized calcium apatite, calcium, phosphate, and hydroxyl ions $\left(\mathrm{Ca}_{10}\left(\mathrm{PO}_{4}\right)_{6}(\mathrm{OH})_{2}\right)$. Biological apatites contain a number of trace ions, such as $\mathrm{Mg}^{2+}, \mathrm{F}^{-}, \mathrm{Cl}^{-}, \mathrm{CO}_{3}{ }^{2-}$, and $\mathrm{HPO}_{4}{ }^{2-}$, in addition to its main components [24]. The most abundant of the minor cations present in bone mineral are $\mathrm{Na}^{+}, \mathrm{Mg}^{2+}$, and $\mathrm{K}^{+}$[25]. The precise location of these elements is uncertain. Some studies suppose that they mainly belong to the apatite lattice, but others are of the opinion that they are simply adsorbed on the surface of crystallites [26]. The exchange and migration activity of these elements is the inherent property of living bone and reflects the state of bone to some extent. The surface of nanocrystals plays an exceptional role as a boundary between mineral and organic components, determining some biological processes. The water molecules adsorbed on the surfaces of apatite crystals (structured hydrated layer) stabilize them and serve as an environment for mobile ions, providing mass transfer and promoting the development of apatite domains [27]. To date, the effects of ICA on the mineral phase of bone have not been elucidated.

Here, we investigated the effects of ICA on bone loss in a hindlimb suspension rodent model and found that ICA treatment significantly attenuated microgravity-induced bone loss by suppressing bone resorption as well as adjusting the distribution of apatite ions in bone.

\section{MATERIALS AND METHODS}

Animals

Two-month-old female Wistar rats, with a body weight of $191 \pm 10$ $\mathrm{g}$, were purchased from Lanzhou University (Lanzhou, China) and acclimatized for 1 week in an air-conditioned room at a temperature of $23 \pm 2{ }^{\circ} \mathrm{C}$ with $12 \mathrm{~h} / 12 \mathrm{~h}$ light-dark illumination cycles and humidity $45-50 \%$. The rats were randomly divided into three groups ( $n=6$ in each group), including the control (Ctrl) group, the hindlimb suspension with vehicle (HLS) group, and the hindlimb suspension with ICA (HLS + ICA) group. The Ctrl group was treated with vehicle $(0.9 \%$ saline $)$ but without hindlimb suspension, while both the HLS group and the ICA group were hindlimb unloaded by suspending their tails according to the recommendation of Morey-Holton and Globus [7], and orally administered a vehicle or $25 \mathrm{mg} \cdot \mathrm{kg}^{-1} \cdot \mathrm{d}^{-1}$ of ICA (purity $>98 \%$,
Herbest, Baoji, China), respectively. The dosage was chosen based on previous studies [28-30]. All rats were provided with standard laboratory rodent chow and water ad libitum. During the 4 weeks of administration, the general situation of eating, drinking, activity, color gloss, and body weight of the rats were closely observed and recorded. All procedures used were approved by the Institutional Animal Care and Use Committee at the School of Pharmacy of Lanzhou University.

Tissue collection and sample preparation

The rats fasted for $24 \mathrm{~h}$ after the last administration. The next day, the rats were anesthetized with $10 \%$ chloral hydrate, and whole blood was collected by abdominal aorta puncture at the end of the laparotomy. After the blood was left standing for $3 \mathrm{~h}$, the collected blood was centrifuged at $1500 \times g$ for 15 min to acquire the supernatant. The serum was dispensed into small tubes $(20 \mu \mathrm{L})$ and stored in a freezer at $-80^{\circ} \mathrm{C}$ for later use. The femora and tibiae were cleared from the attached muscle and collected. The left femora were rinsed with saline and packed with salineinfiltrated medical gauze and placed in the freezer at $-20^{\circ} \mathrm{C}$ for later BMD testing, biomechanics analysis, and mobile ionic measurement. The right femora were immersed in $75 \%$ alcohol and stored in the freezer at $-20^{\circ} \mathrm{C}$ for micro-CT analysis. The tibiae were wrapped in medical gauze and stored in liquid nitrogen for the analysis of alkaline phosphatase (ALP), bone glaprotein (BGP), osteoprotegerin (OPG), and receptor activator of the NF-KB ligand (RANKL) mRNA expression.

\section{BMD analysis and biomechanical testing}

Total BMD of the femora from hindlimbs was measured by dualenergy X-ray absorptiometry (GE Healthcare, Madison, USA) for the assessment using the small laboratory animal mode. Total femoral BMD was calculated using enCORE software (GE Healthcare). Three-point bending tests were performed using a precision universal tester (SHMADZU, Kyoto, Japan). Each bone specimen was placed on two supports $15 \mathrm{~mm}$ apart, and a load was applied to the middle position of the femur at a rate of 10 $\mathrm{mm} / \mathrm{min}$ until the specimen was fractured. The biomechanical parameters, including the maximal force $(\mathrm{N})$ and elasticity modulus $\left(\mathrm{N} / \mathrm{mm}^{2}\right)$, of bone specimens were recorded.

\section{Micro-CT analysis}

The distal metaphysis of the femur from the hindlimbs was scanned using a Skyscan 1176 high-resolution micro-CT (Bruker, Kontich, Belgium). Three-dimensional (3D) image data were obtained using the CT-analyzer software (Bruker) at $14-\mu \mathrm{m}$ resolution, and the regions from the epiphyseal line to $2 \mathrm{~mm}$ below were chosen to provide data for the analysis. Direct 3D measurement methods were used to calculate the following parameters of trabecular bone: the BMD of cancellous bone (cancellous BMD; $\mathrm{mg} / \mathrm{cm}^{3}$ ), trabecular bone volume fraction (BV/ TV; \%), trabecular thickness (Tb.Th; mm), trabecular number (Tb.N; $1 \mathrm{~mm}$ ), trabecular separation (Tb.Sp; $\mathrm{mm}$ ), and structure model index (SMI).

\section{ELISA}

The cryopreserved sera were brought to room temperature to thaw. After centrifugation for $5 \mathrm{~min}$ at $150 \times g$, the concentration of $\mathrm{Ca}^{2+}$ and the bone resorption marker, tartrate-resistant acid phosphatase-5b (TRACP-5b), in serum were measured using ELISA kits (Mlbio, Shanghai, China) according to the manufacturer's instructions.

RNA extraction and qRT-PCR

Total RNA of the tibia was extracted using TRlzol reagent (Invitrogen, Carlsbad, USA). Reverse transcription was performed using the All-in-One ${ }^{\mathrm{TM}}$ First-Strand cDNA Synthesis Kit (GeneCopoeia, Guangzhou, China). To quantify ALP, BGP, OPG, and RANKL 
mRNA expression, real-time PCR was performed using the All-inOne $^{\mathrm{TM}}$ mRNA qPCR Detection Kit (GeneCopoeia) based on SYBR Green. GAPDH was used to normalize the relative amount of mRNA. All of the primers were purchased from GeneCopoeia. The $2^{-\triangle \triangle \mathrm{Ct}}$ method was used to calculate the relative gene expression.

X-ray diffraction analysis

The femur was treated using a method described previously [31], and X-ray diffraction (XRD) investigations were performed using the diffractometer DRON4-07 (Burevestnik, Russia) connected to a computer-aided experiment control and data processing system. CoKa radiation was used with a conventional Bragg-Brentano $\theta-2 \theta$ geometry ( $\theta$ is Bragg's angle). The current and voltage of the $\mathrm{X}$-ray tube were $20 \mathrm{~mA}$ and $30 \mathrm{kV}$, respectively. The bone samples were measured in the continuous registration mode (at the speed of $1.0^{\circ} / \mathrm{min}$ ) within the $2 \theta$ angle range from $10^{\circ}$ to $70^{\circ}$. All data processing procedures were conducted with the use of the program package DIFWIN-1 (Etalon-PTC, Russia). Phase analysis was performed by comparing the diffraction patterns from the

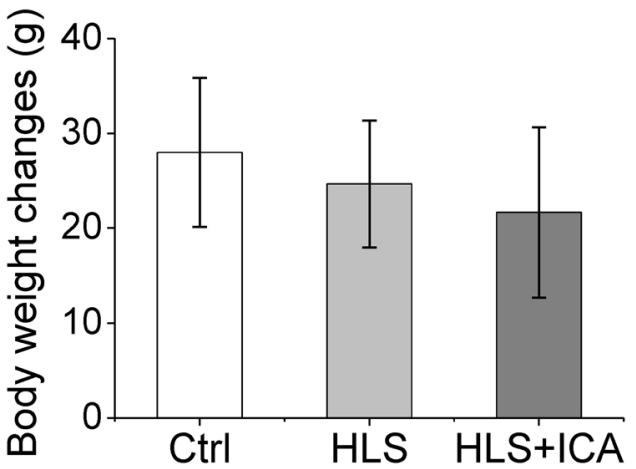

Fig. 2 Body weight changes in the different groups. Ctrl control group, HLS hindlimb suspension group, HLS + ICA hindlimb suspension and treated with icariin group. Data are shown as the mean $\pm S D, n=6$ in each group investigated samples and the reference data JCPDS. To evaluate the phase purity and stoichiometry of biological apatite, the diaphysis of the femur was examined in the initial state and after annealing in the electric furnace at the temperature of $950^{\circ} \mathrm{C}$ for $1 \mathrm{~h}$.

Mobile ionic species measurement

The mobile ionic species were analyzed using atomic spectrometry (AS) as previously described [32]. In brief, the small separate portions of the grinded diaphysis of the femur were heated in an electric furnace at the $600-760^{\circ} \mathrm{C}$ range with a $40^{\circ} \mathrm{C}$ step increment; each temperature was kept for $1 \mathrm{~h}$. After annealing, the powdered material was treated with ultrasound (15-20 W/ $\mathrm{cm}^{2}, 22 \mathrm{kHz}$ ) for 10-15 min in distilled water in a UZDN-A device (Selmi, Ukraine). The suspension obtained was filtered, and the concentration of the elements was measured in liquid using the atomic spectrometer KAS 120.1 (Selmi, Ukraine). The analytical system includes a double-beam spectrophotometer equipped with a deuterium device for background absorption correction. $\mathrm{Mg}^{2+}$ was determined using flame atomic absorption spectroscopy, and $\mathrm{Na}^{+}$and $\mathrm{K}^{+}$by flame atomic emission spectroscopy; in both cases, an air-acetylene flame was used.

\section{Statistical analysis}

Statistical analysis was performed using SPSS 19.0 software (SPSS, Chicago, USA). Data were analyzed using a one-way ANOVA and followed by Tukey's multiple comparison test as a post test to compare the group means if the overall $P<0.05$. All data are expressed as the mean \pm SD. A level of $P<0.05$ was considered statistically significant.

\section{RESULTS}

The general effect of ICA treatment in rats

The rats in all groups displayed normal eating, drinking, activity, and coat color. As shown in Fig. 2, after administration for 4 weeks, the body weight changes of the HLS group and the ICA group were lower than that of the Ctrl group; however, the difference was not statistically significant.

a

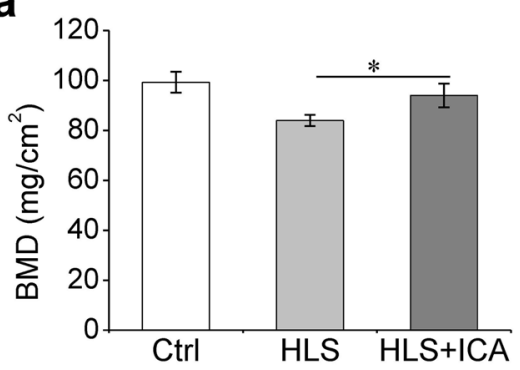

b

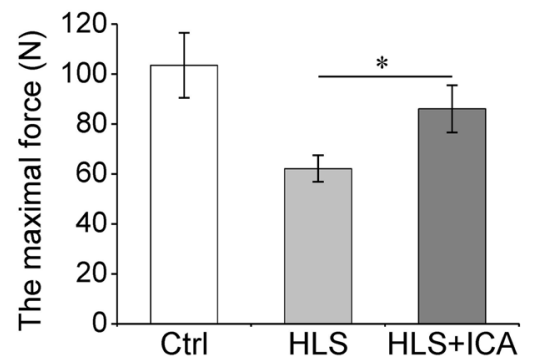

C

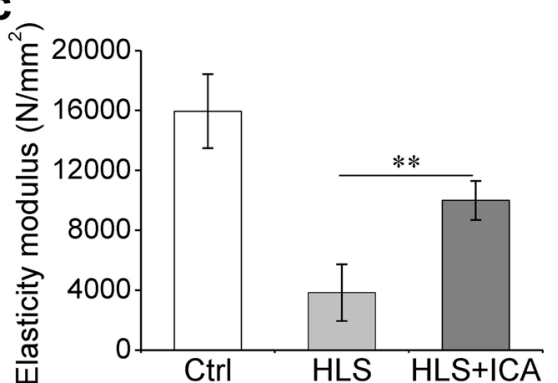

Fig. 3 The bone mineral density (BMD) and mechanical parameters of hindlimb bones. a Total femur BMD assessed using dual-energy X-ray absorptiometry; b, c Mechanical parameters of the bone samples from three-point bending tests; $\mathbf{b}$ the maximal force (N); $\mathbf{c}$ Elasticity modulus $\left(\mathrm{N} / \mathrm{mm}^{2}\right)$; Ctrl control group, HLS hindlimb suspension group, HLS + ICA hindlimb suspension and treated with icariin group. Data are shown as the mean $\pm S D, n=6$ in each group. ${ }^{*} P<0.05,{ }^{* *} P<0.01$ vs the HLS group 
a

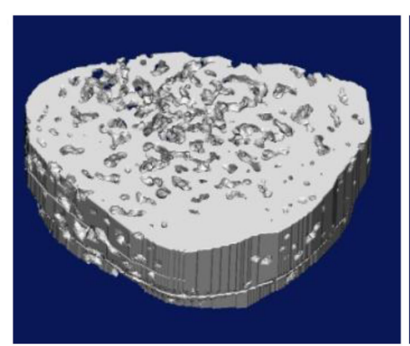

Ctrl

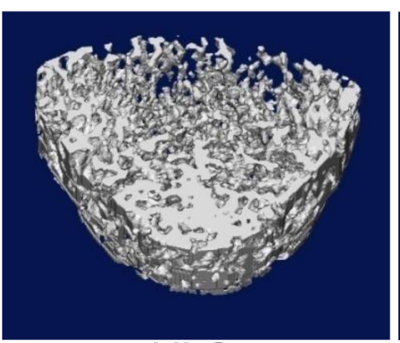

HLS

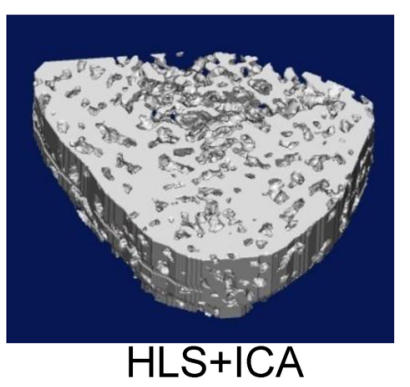

HLS+ICA b

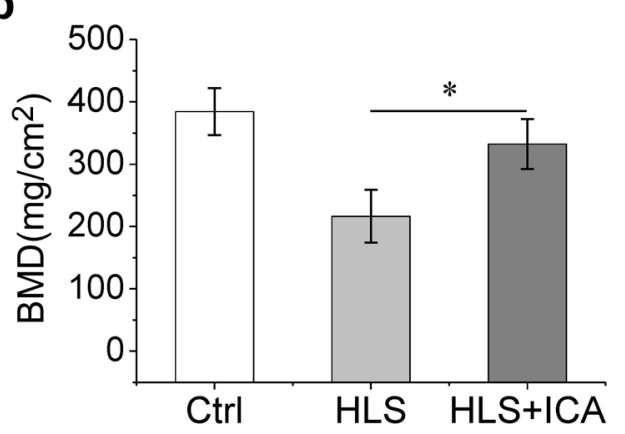

d

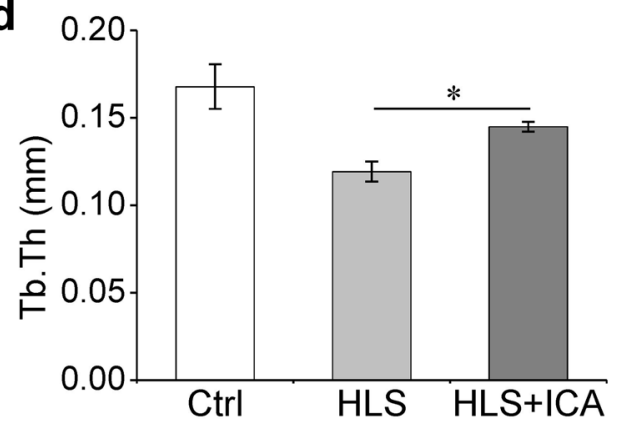

f

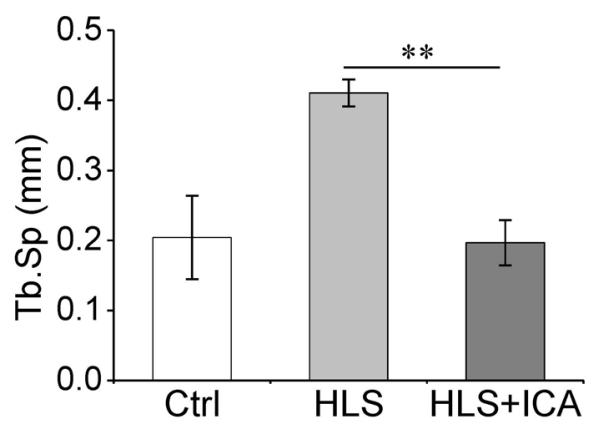

C

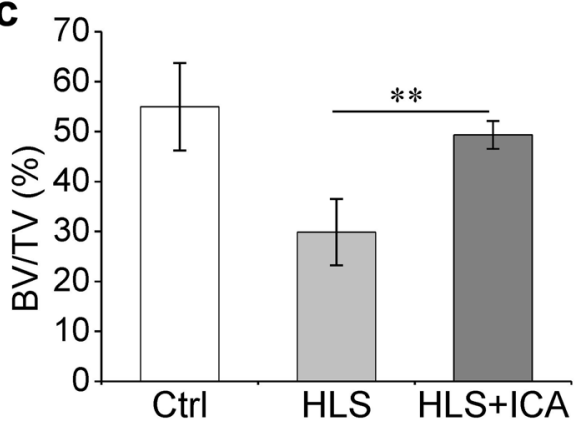

e

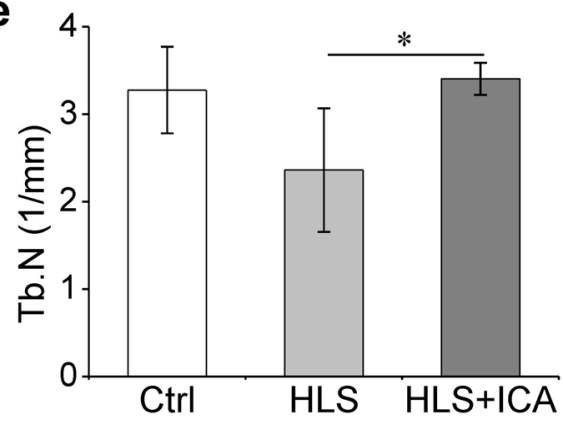

Fig. 4 ICA treatment exhibits reduced damage of cancellous bone induced by hindlimb suspension. a Representative micro-CT reconstructive images of the distal femurs of the Ctrl, HLS, and HLS + ICA rats; $\mathbf{b}-\mathbf{g}$ The bone micro-architectural parameters; BV/TV trabecular bone volume fraction, Tb.Th trabecular thickness, Tb.Sp trabecular separation, Tb.N trabecular number, SMI structure model index. Ctrl control group, HLS hindlimb suspension group, HLS + ICA hindlimb suspension and treated with icariin group. Data are shown as the mean \pm SD, $n=6$ in each group. ${ }^{*} P<0.05,{ }^{* *} P<0.01$ vs the HLS group

ICA treatment rescues bone loss induced by HLS

To investigate the effects of ICA on the bone mass change induced by simulated microgravity, the femoral BMD and mechanical parameters of the hindlimbs were measured. As shown in Fig. 3a, hindlimb suspension led to significant bone mass loss; the femoral BMD decreased from $99.29 \pm 4.20 \mathrm{mg} / \mathrm{cm}^{2}$ in the Ctrl group to $84.02 \pm 2.23 \mathrm{mg} / \mathrm{cm}^{2}$ in the HLS group. However, following ICA administration for 4 weeks, the femoral BMD increased by $\sim 12 \%$ compared with that of the HLS group ( $P=$
0.016). Additionally, the three-point bending test results showed that hindlimb suspension remarkably impaired the bone strength of the femur. Compared with the Ctrl group, the ultimate force and elasticity modulus of the femur decreased by $38 \%$ and $67 \%$ in the HLS group, respectively. These two mechanical parameters were significantly recovered in the hindlimb suspension rats treated with ICA (Fig. 3b, c).

Furthermore, micro-CT analyses were performed to explore the microstructure changes in the hindlimb bones. Compared with 

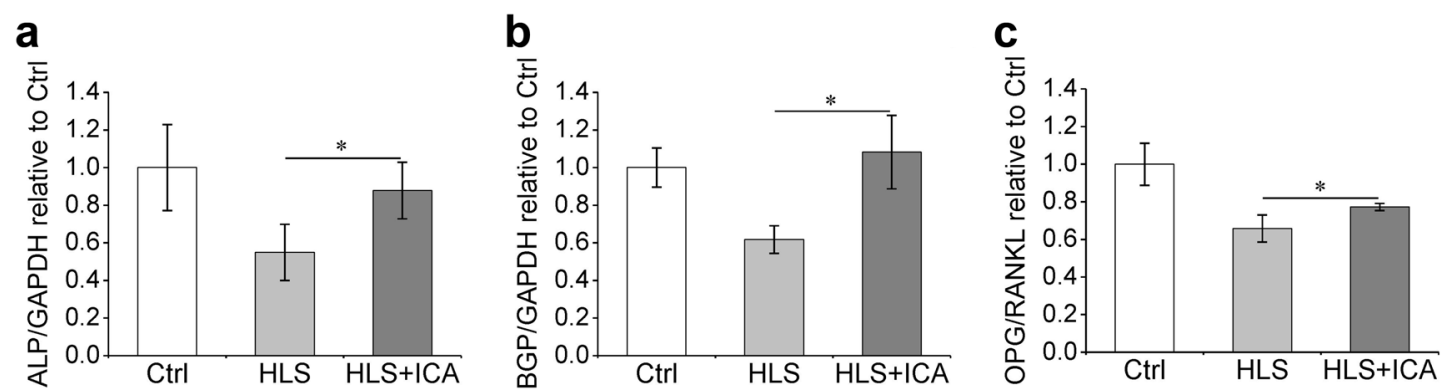

Fig. 5 ICA treatment facilitates osteoblast activity and suppresses osteoclast activity in hindlimb suspension rats. The relative expression of osteoblast differentiation related genes ALP (a), BGP (b) and the ration of OPG/RANKL (c) was evaluated using qRT-PCR. Total RNA was extracted from the tibia. GAPDH was used to normalize the gene expressions and the results are presented as the relative expression compared with the Ctrl group. Ctrl control group, HLS hindlimb suspension group, HLS + ICA hindlimb suspension and treated with icariin group. Data are shown as the mean $\pm S D, n=6$ in each group; ${ }^{*} P<0.05$ vs the HLS group
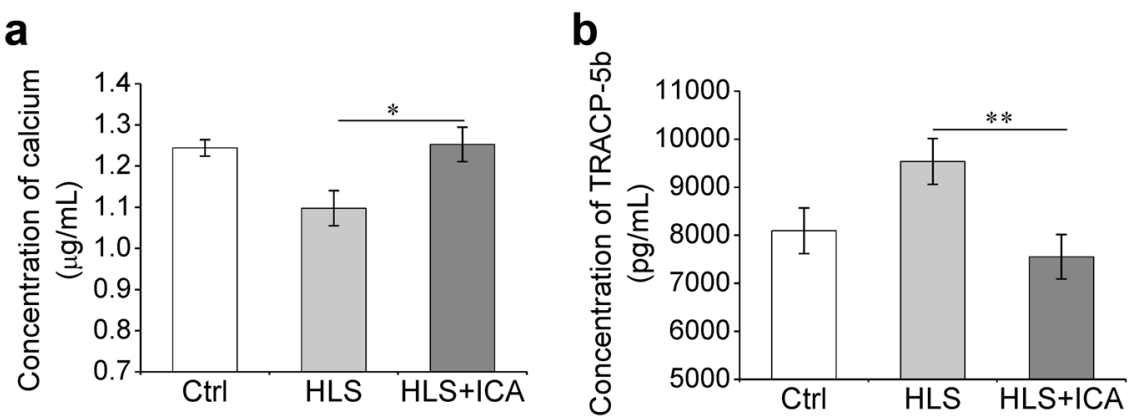

Fig. 6 ICA treatment suppresses bone resorption induced by simulated microgravity. The levels of serum ionic calcium and tartrate-resistant acid phosphatase 5b (TRACP-5b) were measured using ELISA. The concentration of ionic calcium (a) and TRACP-5b (b) were calculated with the standard curves, respectively. Ctrl control group, HLS hindlimb suspension group, HLS + ICA hindlimb suspension and treated with icariin group. Data are shown as the mean $\pm S D, n=6$ in each group. ${ }^{*} P<0.05,{ }^{*} P<0.01$ vs the HLS group

the control group, the hindlimb suspension rats showed lower cancellous BMD, trabecular bone volume fraction (BV/TV), thickness (Tb.Th) and number (Tb.N), and higher trabecular separation (Tb.Sp), and SMI (Fig. 4). As expected, supplementation with ICA notably reversed the changes in these parameters induced by hindlimb suspension (Fig. 4). Taken together, these results indicated that ICA treatment efficiently attenuated the bone mass loss and trabecular bone architecture damage induced by hindlimb suspension.

ICA treatment suppresses osteoclast activity in HLS rats As the above data have revealed the role of ICA in the maintenance of bone mass, we speculated that ICA would improve the osteogenesis in HLS rats. To validate this speculation, the mRNA expression levels of osteoblast-related genes, such as $A L P, B G P$, and OPG, and the osteoclast-related gene RANKL in the tibia from hindlimbs were analyzed using qRT-PCR assay. As shown in Fig. 5, the expression of ALP and BGP in the HLS group was downregulated by approximately twofold compared with that in the Ctrl group. Moreover, the ratio of OPG/RANKL was also decreased, which indicates the increased activity of bone resorption. However, the treatment of ICA dramatically upregulated the levels of ALP, BGP, and the OPG/RANKL ratio in the hindlimb suspension rats. These results indicated that ICA treatment suppressed osteoclast activity and facilitated the osteoblast activity to some extent in the HLS rats.

ICA treatment recovers the levels of bone metabolism markers in serum

The biochemical markers of bone metabolism in serum were measured using ELISA. As shown in Fig. 6a, the concentration of $\mathrm{Ca}^{2+}$ in the HLS group was greatly lower than that in the Ctrl group, whereas it was restored to normal levels when ICA was administered $(P=0.03)$. On the contrary, the concentration of TRACP-5b, a bone absorption biochemical index in serum, obviously increased by $17.8 \%$ in the hindlimb suspension rats compared with that in the control rats and decreased by $20.9 \%$ when administered with ICA compared with that in the HLS group $(P=0.009$, Fig. 6b). This result indicated that ICA treatment suppressed the activity of bone resorption induced by hindlimb suspension.

ICA treatment suppresses HLS-induced instability of the bone mineral content

The bone XRD patterns of all bone samples after annealing showed the presence of crystalline apatite as a major phase and $\beta$ tricalcium magnesium phosphate ( $\beta$-TCMP) as a minor phase after annealing at $950^{\circ} \mathrm{C}$. The most suitable reference phase of the $\beta$ TCMP family $\left(\mathrm{Ca}_{3-x} \mathrm{Mg}_{x}\left(\mathrm{PO}_{4}\right)_{2}\right)$ was found to be $\mathrm{Ca}_{2,86} \mathrm{Mg}_{0,14}\left(\mathrm{PO}_{4}\right)_{2}$ (JCPDS 77-692). In spite of a low concentration of $\beta$-TCMP, the arrangement of all groups exhibited $C_{\beta-T C M P}^{\mathrm{I}} \geq \mathrm{C}_{\beta-\mathrm{TCMP}}^{\mathrm{C}}>\mathrm{C}_{\beta \text {-TCMP }}^{\mathrm{H}}$ $\left(C_{\beta \text {-TCMP: }}^{X}\right.$ the concentration of $\beta$-TCMP in wt \%; X: ICA/Ctrl/HLS group) as shown in Fig. 7a. Other minor phases were not found. These results indicate that hindlimb suspension reduced the ability of bone apatite to perform lattice substitution, especially the co-incorporation of sodium and carbonate.

As it was shown in an earlier publication [33], in the $600-760^{\circ} \mathrm{C}$ range, a high mobility of some ions in bone mineral was observed. We further analyzed the concentration of $\mathrm{Na}^{+}, \mathrm{Mg}^{2+}$, and $\mathrm{K}^{+}$in water after ultrasonic treatment of the annealed bone. As represented in Fig. $7 \mathrm{~b}-\mathrm{d}$, the concentrations of $\mathrm{Na}^{+}, \mathrm{Mg}^{2+}$, and $\mathrm{K}^{+}$in the organic phase (plus bulk water) were considerably higher for the HLS group vs the Ctrl group. On the contrary, the concentrations of $\mathrm{Na}^{+}, \mathrm{Mg}^{2+}$, and $\mathrm{K}^{+}$were lower in the HLS group 
a

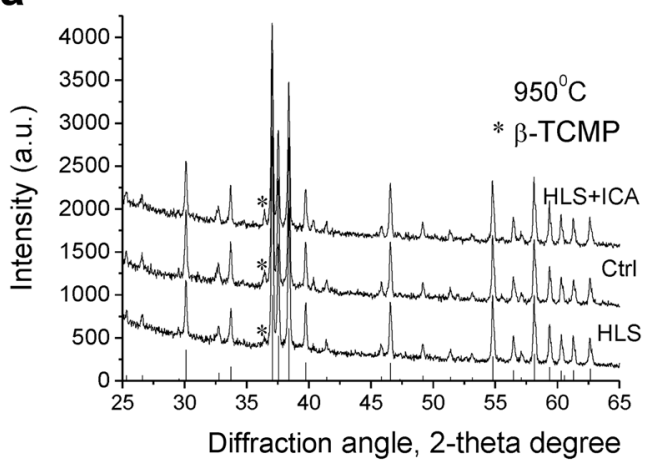

C

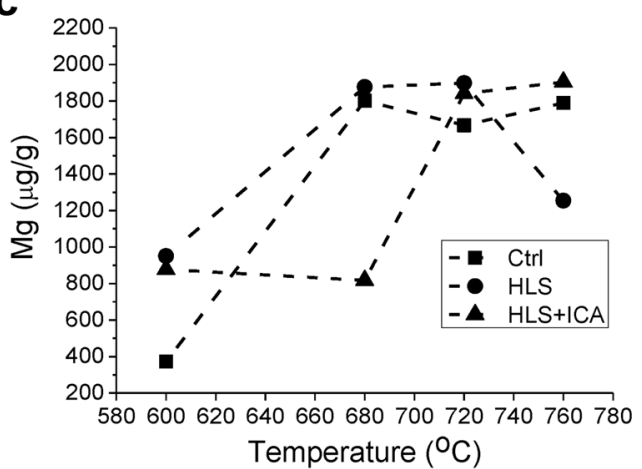

b

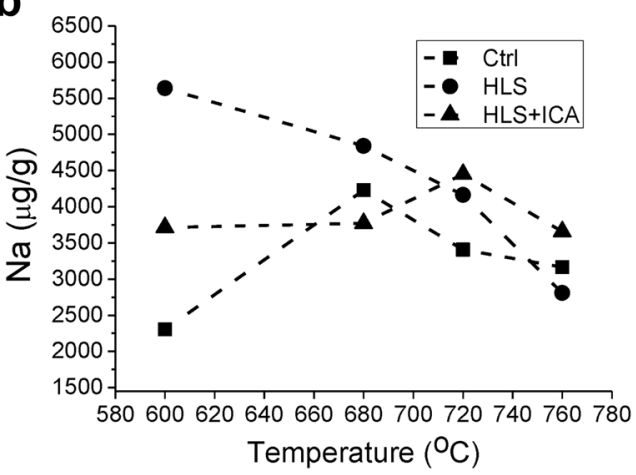

d

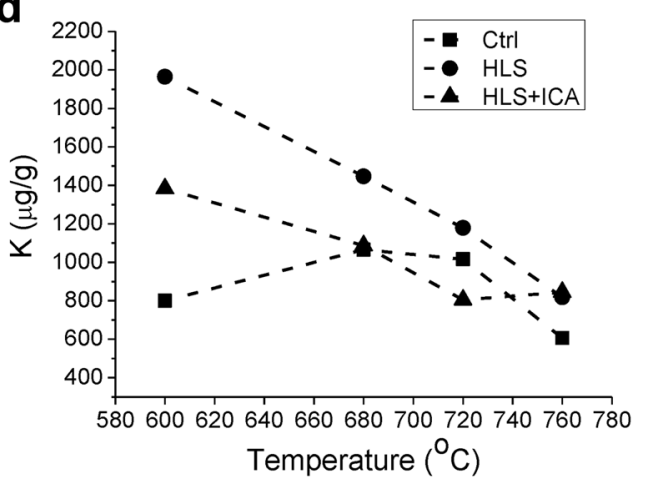

Fig. 7 ICA treatment attenuates the instability of the bone mineral phase induced by hindlimb suspension. a X-ray diffraction patterns of bone samples after the annealing at $950{ }^{\circ} \mathrm{C}$ for $1 \mathrm{~h}$; ${ }^{*}$ indicates the peak of $\beta$-TCMP; $\mathbf{b}$ - $\mathbf{d}$ concentrations of $\mathrm{Na}^{+}(\mathbf{b}), \mathrm{Mg}^{2+}(\mathbf{c})$, and $\mathrm{K}^{+}(\mathbf{d})$ in water medium after ultrasonic treatment of the annealed and powdered bone samples. The lines are drawn only to guide the eye. Ctrl control group, HLS hindlimb suspension group, HLS + ICA hindlimb suspension and treated with icariin group

to some extent when ICA was applied. Taken together, these data indicated that ICA prevented simulated microgravity-induced bone loss via enhancing the stability of bone apatite and reducing the metabolic disorder of bone mineral.

\section{DISCUSSION}

ICA has been shown to prevent osteoporosis in postmenopausal women and ovariectomized rats [17, 34]. In our investigation, we systematically evaluated its preventive effect on bone loss in rats treated with simulated microgravity. The obtained data showed that ICA remarkably reduced bone mass loss and enhanced bone strength in the hindlimb suspension rodent model. Treatment with ICA not only suppressed the activity of bone resorption, but enhanced the stability of bone apatite and reduced the exchange of mobile ions.

Bone strength depends on BMD, trabecular bone architecture, connectivity, and mineralization $[35,36]$. Our data revealed that hindlimb suspension significantly reduced the bone maximal force and elasticity modulus, indicating a decrease in bone strength. The micro-CT results showed that treatment with ICA restored the HLS induced-damage to trabecular bone architecture via increases in BV/TV, Tb.Th, and Tb.N and a decrease in the Tb.Sp parameter. In addition, the XRD results demonstrated that the calcium apatite of the bone mineral phase was stabilized by ICA treatment. All the results suggest that ICA treatment exhibits beneficial effects on bone mass and strength in the hindlimb suspension rats through stabilizing the trabecular bone architecture and bone apatite mineralization.

Biochemical parameters in serum are of great value as indicators for osteoporosis diagnosis and effectiveness evaluation of clinical treatments $[37,38]$. We measured the expression of the bone resorption marker TRACP-5b in serum [39]. Our data showed that the concentration of serum TRACP-5b in the ICA treatment group was significantly lower than that in the HLS group, indicating an effective suppression of bone resorption by ICA. Moreover, it has been in agreement that calcium is an essential component of anti-resorptive agent therapy for osteoporosis [40]. Previous studies discovered that serum calcium presented ascendant change during a short-term hindlimb suspension in rats [41, 42]. However, its expression levels were found to be downregulated in postmenopausal women or ovariectomized rats $[43,44]$. Our data showed that the concentration of serum calcium and the ratio of OPG/RANKL were substantially lower in long-term hindlimb suspension rats compared with that in control rats. Additionally, ICA administration significantly recovered the calcium levels in serum and the ratio of OPG/RANKL in bone cells, which are consistent findings with the results obtained from ovariectomized rats [44]. Collectively, our results indicate that ICA recovers the simulated microgravity-induce bone resorption and calcium metabolic disorder.

The XRD patterns validated the presence of poorly crystalline apatite in all groups. Noteworthy, the XRD data of the initial bone were not of sufficient resolution to allow quantitative characterization of the mineral; for example, it was impossible to determine the size of the apatite unit cell with sufficient accuracy or to detect the traces of other phases. Further elevation in temperature caused a high mobility of some ions in bone mineral due to recrystallization of the bioapatite accompanied by the elimination of $\mathrm{CO}_{3}{ }^{2-}$ ions [45]. However, when the temperature was higher than $760^{\circ} \mathrm{C}$, a reduction of the labile species indicates that the earlier available anions and cations formed electrically neutral and insoluble compounds during heating or/and entered into apatite. For example, $\mathrm{Mg}^{2+}$ participates in the formation of $\beta$-TCMP. Normally the thermal behavior of labile $\mathrm{Na}^{+}$has a non-monotonic character in the range from 600 to $760{ }^{\circ} \mathrm{C}$, but the labile $\mathrm{Na}^{+}$ 
monotonously decreases. Therefore, we hypothesize that unusual thermal behavior of labile $\mathrm{Na}^{+}$in the HLS group can be explained by the low ability of their bioapatite to trap carbonates and create $\mathrm{CO}_{3}{ }^{2-} \rightarrow \mathrm{PO}_{4}{ }^{3-}$ substitution. In turn, the reduced ability to accept the carbonate ion could prevent progressive dissolution of apatite crystals $[46,47]$ and maintain their stability. In addition, the labile $\mathrm{K}^{+}$exhibited the same trend in the HLS group; however, it should be noted that $\mathrm{K}^{+}$was not able to join the apatite structure in a significant amount but was present rather as a surface species and in a surrounding medium due to its rather large size, such as in the labile state. Normally concentrations of labile $\mathrm{K}^{+}$do not change significantly under heating conditions until the formation of insoluble compounds at a higher temperature. When treated with ICA, the labile $\mathrm{Na}^{+}$and $\mathrm{K}^{+}$revealed some similarities to the Ctrl group, which indicates that ICA mitigated changes in the bioapatite crystals and has a positive influence on the stabilization of the bone mineral in hindlimb suspension rats.

In conclusion, our data provide evidence that ICA prevents bone loss in the hindlimb suspension rodent model at least partly through mediation of the balance between bone formation and resorption. Notably, our results also reveal that ICA treatment improves bone apatite stability in simulated microgravity. Therefore, this study is able to shed light on the potential application of Chinese medicine ingredients as pharmacological countermeasures for bone loss in long-duration spaceflight.

\section{ACKNOWLEDGEMENTS}

This work was supported by grants from the International Science \& Technology Cooperation Program of China (ISTCP, No. 2015DFR30940), the Chinese Academy of Science (CAS) "Light of West China" Program (2015), Special Program from Chinese Academy of Science in Cooperation with Russia, Ukraine and the Republic of Belarus $(2015,2017)$, the Introduced Intelligence Project from the State Administration of Foreign Experts Affairs P.R. China (2016), and the Science and Technology Research Project of Gansu Province (Nos. 145RTSA012 and 17JR5RA307).

\section{AUTHOR CONTRIBUTIONS}

J-fW and J-pH designed the study; XF, J-pH, and W-gS performed the animal experiments; SD and AK contributed the XRD and mobile ionic species analysis; $H L$ and $M Z$ assisted in caring for the animals; $X F, J-p H$, and SD performed the data analyses; J-pH, XF, and J-fW wrote the manuscript.

\section{ADDITIONAL INFORMATION}

Competing interests: The authors declare no competing interests.

\section{REFERENCES}

1. Thirsk R, Kuipers A, Mukai C, Williams D. The space-flight environment: the International Space Station and beyond. CMAJ. 2009;180:1216-20.

2. Lang T, LeBlanc A, Evans H, Lu Y, Genant H, Yu A. Cortical and trabecular bone mineral loss from the spine and hip in long-duration spaceflight. J Bone Miner Res. 2004;19:1006-12.

3. Kondo H, Yumoto K, Alwood JS, Mojarrab R, Wang A, Almeida EA, et al. Oxidative stress and gamma radiation-induced cancellous bone loss with musculoskeletal disuse. J Appl Physiol. 2010;108:152-61.

4. Ya-Jing Y, Da-Chuan Y, Peng S. Effect of microgravity and a high magnetic field on hydroxyapatite deposition and implications for bone loss in space. Appl Surf Sci. 2010;256:7535-9.

5. Schwarzenberg M, Pippia P, Meloni MA, Cossu G, Cogoli-Greuter M, Cogoli A. Microgravity simulations with human lymphocytes in the free fall machine and in the random positioning machine. J Gravit Physiol. 1998;5:P23-6.

6. Hargens AR, Vico L. Long-duration bed rest as an analog to microgravity. J Appl Physiol. 2016;120:891-903.

7. Morey-Holton ER, Globus RK. Hindlimb unloading rodent model: technical aspects. J Appl Physiol. 2002;92:1367-77.

8. Convertino VA. Exercise as a countermeasure for physiological adaptation to prolonged spaceflight. Med Sci Sports Exerc. 1996;28:999-1014.

9. Cavanagh PR, Licata AA, Rice AJ. Exercise and pharmacological countermeasures for bone loss during long-duration space flight. Gravit Space Biol Bull. 2005; 18:39-58.
10. Ettinger B, Genant HK, Cann CE. Postmenopausal bone loss is prevented by treatment with low-dosage estrogen with calcium. Ann Intern Med. 1987;106:40-5.

11. Reginster JY. The role of bisphosphonates in the prevention and treatment of osteoporosis. Clin Rheumatol. 1995;14 (Suppl 3):22-5.

12. Rizzoli R, Reginster JY, Boonen S, Breart G, Diez-Perez A, Felsenberg D, et al. Adverse reactions and drug-drug interactions in the management of women with postmenopausal osteoporosis. Calcif Tissue Int. 2011;89:91-104.

13. Rossouw JE, Anderson GL, Prentice RL, LaCroix AZ, Kooperberg C, Stefanick ML, et al. Risks and benefits of estrogen plus progestin in healthy postmenopausal women: principal results From the Women's Health Initiative randomized controlled trial. JAMA. 2002;288:321-33.

14. Bolland MJ, Grey A, Avenell A, Gamble GD, Reid IR. Calcium supplements with or without vitamin D and risk of cardiovascular events: reanalysis of the Women's Health Initiative limited access dataset and meta-analysis. BMJ. 2011;342:d2040.

15. Li C, Li Q, Mei Q, Lu T. Pharmacological effects and pharmacokinetic properties of icariin, the major bioactive component in Herba Epimedii. Life Sci. 2015;126:57-68.

16. Zhang DC, Liu JL, Ding YB, Xia JG, Chen GY. Icariin potentiates the antitumor activity of gemcitabine in gallbladder cancer by suppressing NF-kappaB. Acta Pharmacol Sin. 2013;34:301-8.

17. Zhang G, Qin L, Shi Y. Epimedium-derived phytoestrogen flavonoids exert beneficial effect on preventing bone loss in late postmenopausal women: a 24month randomized, double-blind and placebo-controlled trial. J Bone Miner Res. 2007;22:1072-9.

18. Chen G, Wang C, Wang J, Yin S, Gao H, Xiang LU, et al. Antiosteoporotic effect of icariin in ovariectomized rats is mediated via the $\mathrm{Wnt} / \beta$-catenin pathway. Exp Ther Med. 2016;12:279-87.

19. Huang J, Yuan L, Wang X, Zhang TL, Wang K. Icaritin and its glycosides enhance osteoblastic, but suppress osteoclastic, differentiation and activity in vitro. Life Sci. 2007;81:832-40.

20. Zhao J, Ohba S, Shinkai M, Chung UI, Nagamune T. Icariin induces osteogenic differentiation in vitro in a BMP- and Runx2-dependent manner. Biochem Biophys Res Commun. 2008;369:444-8.

21. Chen KM, Ge BF, Liu XY, Ma PH, Lu MB, Bai MH, et al. Icariin inhibits the osteoclast formation induced by RANKL and macrophage-colony stimulating factor in mouse bone marrow culture. Pharmazie. 2007;62:388-91.

22. LeGeros RZ. Properties of osteoconductive biomaterials: calcium phosphates. Clin Orthop Relat Res. 2002;395:81-98.

23. Zioupos P, Currey JD, Hamer AJ. The role of collagen in the declining mechanical properties of aging human cortical bone. J Biomed Mater Res. 1999;45:108-16.

24. Bigi A, Foresti E, Gregorini R, Ripamonti A, Roveri N, Shah JS. The role of magnesium on the structure of biological apatites. Calcif Tissue Int. 1992;50:439-44.

25. Elliott JC, Anderson P, Gao XJ, Wong FS, Davis GR, Dowker SE. Application of scanning microradiography and $x$-ray microtomography to studies of bones and teeth. J X-ray Sci Technol. 1994;4:102-17.

26. Rey C. Calcium phosphate biomaterials and bone mineral. Differences in composition, structures and properties. Biomaterials. 1990;11:13-5.

27. Rey C, Combes C, Drouet C, Sfihi H, Barroug A. Physico-chemical properties of nanocrystalline apatites: Implications for biominerals and biomaterials. Mater Sci Eng: C. 2007;27:198-205.

28. Cheng K, Baofeng GE, Chen K, Zhen P, Zhou J, Xiaoni MA, et al. Oral medication of icariin enhances peak bone mineral density and bone quality in rats. Chin J Osteoporos. 2014;20:120-4.

29. Cheng K, Chen KM, Ge BF, Zhen P, Ma HP, Gao YH. Effect of icariin and genistein on bone protection. Chin Pharmacol Bull. 2014;30:1315-9.

30. Sun P, Liu Y, Deng X, Yu C, Dai N, Yuan X, et al. An inhibitor of cathepsin K, icariin suppresses cartilage and bone degradation in mice of collagen-induced arthritis. Phytomedicine. 2013;20:975-9.

31. Mkukuma LD, Skakle JM, Gibson IR, Imrie CT, Aspden RM, Hukins DW. Effect of the proportion of organic material in bone on thermal decomposition of bone mineral: an investigation of a variety of bones from different species using thermogravimetric analysis coupled to mass spectrometry, high-temperature Xray diffraction, and Fourier transform infrared spectroscopy. Calcif Tissue Int. 2004;75:321-8.

32. Danilchenko SN, Koropov AV, Protsenko IY, Sulkio-Cleff B, Sukhodub LF. Thermal behavior of biogenic apatite crystals in bone: an X-ray diffraction study. Cryst Res Technol. 2006;41:268-75.

33. Danil'chenko SN, Kulik AN, Pavlenko PA, Kalinichenko TG, Bugai AN, Chemeris II, et al. Thermally activated diffusion of magnesium from bioapatite crystals. J Appl Spectrosc. 2006;73:437-43.

34. Li GW, Xu Z, Chang SX, Nian H, Wang XY, Qin LD. Icariin prevents ovariectomyinduced bone loss and lowers marrow adipogenesis. Menopause. 2014;21:1007-16.

35. Einhorn TA. Bone strength: the bottom line. Calcif Tissue Int. 1992:51:333-9. 
36. Muller R, Hannan M, Smith SY, Bauss F. Intermittent ibandronate preserves bone quality and bone strength in the lumbar spine after 16 months of treatment in the ovariectomized cynomolgus monkey. J Bone Miner Res. 2004;19:1787-96.

37. Vasikaran S, Eastell R, Bruyere O, Foldes AJ, Garnero P, Griesmacher A, et al. Markers of bone turnover for the prediction of fracture risk and monitoring of osteoporosis treatment: a need for international reference standards. Osteoporos Int. 2011;22:391-420.

38. Garnero P. New developments in biological markers of bone metabolism in osteoporosis. Bone. 2014;66:46-55.

39. Halleen JM, Tiitinen SL, Ylipahkala H, Fagerlund KM, Vaananen HK. Tartrateresistant acid phosphatase $5 \mathrm{~b}$ (TRACP $5 \mathrm{~b}$ ) as a marker of bone resorption. Clin Lab. 2006;52:499-509.

40. North American Menopause S. The role of calcium in peri- and postmenopausal women: consensus opinion of The North American Menopause Society. Menopause. 2001;8:84-95.

41. Ohmori S, Kanda K, Kawano S, Kambe F, Seo H. Changes in calcium, PTH and 1,25 $(\mathrm{OH}) 2$ vitamin D3 during tail-suspension in ovariectomized rats: effects of estrogen administration. Environ Med. 2000;44:75-8.
42. Ito T, Kurokouchi K, Ohmori S, Kanda K, Murata Y, Izumi R, et al. Changes in serum concentrations of calcium and its regulating hormones during tail suspension in rats. Environ Med. 1996;40:43-6.

43. Okyay E, Ertugrul C, Acar B, Sisman AR, Onvural B, Ozaksoy D. Comparative evaluation of serum levels of main minerals and postmenopausal osteoporosis. Maturitas. 2013;76:320-5.

44. Nian H, Ma MH, Nian SS, Xu LL. Antiosteoporotic activity of icariin in ovariectomized rats. Phytomedicine. 2009;16:320-6.

45. Danilchenko SN. The approach for determination of concentration and location of major impurities $(\mathrm{Mg}, \mathrm{Na}, \mathrm{K})$ in biological apatite of mineralized tissues. J NanoElectron Phys. 2013;5:171-93.

46. Rey C, Combes C, Drouet C, Lebugle A, Sfihi H, Barroug A. Nanocrystalline apatites in biological systems: characterisation, structure and properties. Materwiss Werksttech. 2007;38:996-1002.

47. Elliott JC. Structure and chemistry of the apatites and other calcium orthophosphates. Studies in inorganic chemistry. 1994;18:1-371 